\title{
Triple GEM Tracking Detectors for COMPASS
}

\author{
B. Ketzer, M. C. Altunbas, K. Dehmelt, J. Ehlers, J. Friedrich, B. Grube, S. Kappler, I. Konorov, S. Paul, A. Placci, \\ L. Ropelewski, F. Sauli, L. Schmitt, and F. Simon
}

\begin{abstract}
The small-area tracker of COMPASS, a high-luminosity fixed target experiment at CERN's SPS, includes a set of 20 large-size $\left(31 \times 31 \mathrm{~cm}^{2}\right)$ gas electron multiplier $($ GEM) detectors. Based on gas amplification in three cascaded GEM foils, these devices permit to obtain high gain and good spatial resolution even at very high particle fluxes. A two-coordinate projective readout yields, for each track, highly correlated signal amplitudes on both projections, allowing to resolve multiple hits in high occupancy regions close to the central deactivated area of $5 \mathrm{~cm}$ diameter. At the same time, the material exposed to the beam is minimized. Splitting the amplification in three cascaded stages permits to achieve a gain of $\sim 8000$, necessary for efficient $(>98 \%)$ detection of minimum ionizing particles on both coordinates, already at relatively moderate voltages across individual GEM foils. As a consequence, the probability of a gas discharge to occur when a heavily ionizing particle enters the detector volume, is reduced by more than one order of magnitude at a given gain compared to the initially foreseen double GEM structure. In conjunction with other strategies resulting from extensive $R \& D$ on discharge phenomena, we were able to further reduce both the energy and the probability of such breakdowns. In order to completely exclude permanent damage to the front-end chip by the rare event of a discharge fully propagating to the readout strips, an external electronic protection circuit is used. The operational characteristics of these detectors were examined both in the laboratory and in the beam, where a spatial resolution for minimum ionizing particles of $(46 \pm 3) \mu \mathrm{m}$ and a time resolution of $\sim 15 \mathrm{~ns}$ were achieved. For the 2001 run of COMPASS, a total of 14 triple GEM detectors have been installed. First results from the commissioning phase in the high-intensity $\mu$ beam are presented.
\end{abstract}

Index Terms-Gas detectors, gas electron multiplier, high-rate tracking, position-sensitive particle detectors.

\section{INTRODUCTION}

C OMPASS (COmmon Muon and Proton Apparatus for Structure and Spectroscopy) is a new fixed target experiment at CERN, which came into operation in 2001. It employs high intensity muon and hadron beams $\left(2 \cdot 10^{8} \mu\right.$ per $5 \mathrm{~s}$ spill at $160 \mathrm{GeV} / \mathrm{c}, 10^{8} \mathrm{p}, K, \pi$ per spill at $100-280 \mathrm{GeV} / \mathrm{c}$ ) from the Super Proton Synchrotron (SPS) to investigate the gluon and

Manuscript received December 7, 2001; revised March 27, 2002.

B. Ketzer, A. Placci, L. Ropelewski, and F. Sauli are with CERN, the European Laboratory for Particle Physics, 1211 Genève 23, Switzerland (e-mail: Bernhard.Ketzer@cern.ch).

M. C. Altunbas is with the Department of Physics, SUNY Buffalo, Amherst, NY USA.

K. Dehmelt is with Universität Mainz, 55099 Mainz, Germany.

J. Ehlers is with Universität Heidelberg, 69117 Heidelberg, Germany.

J. Friedrich, B. Grube, I. Konorov, S. Paul, L. Schmitt, and F. Simon are with Technische Universität München, 85748 Garching, Germany.

S. Kappler is with CERN, 1211 Genève 23, Switzerland, and also with Universität Karlsruhe, 76128 Karlsruhe, Germany.

Digital Object Identifier 10.1109/TNS.2002.803891

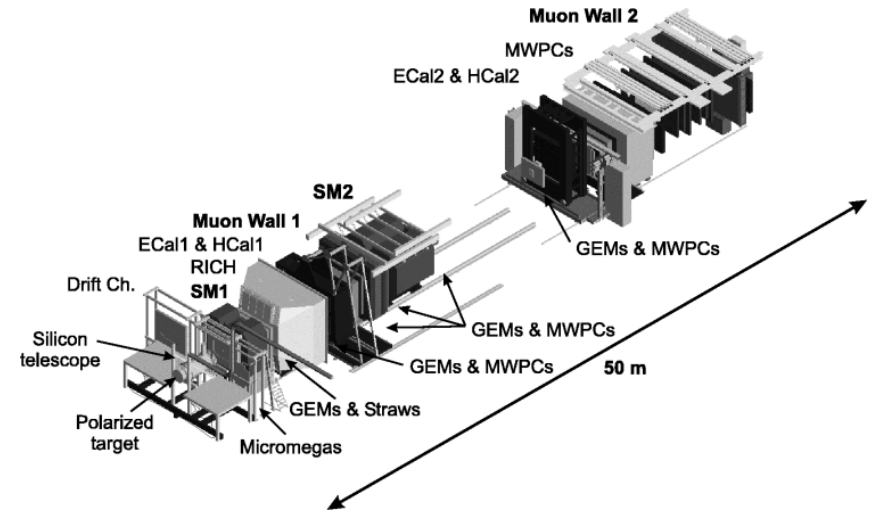

Fig. 1. The COMPASS spectrometer at CERN's SPS accelerator.

quark structure of hadrons [1]. The first goal of the experiment is to measure the gluon contribution to the nucleon spin from the cross-section asymmetry for open charm production in the photon-gluon fusion process [2]. Here, the main focus is on the identification of $D^{0}$ and $\vec{D}^{0}$ mesons by their 2-body decays into $\pi^{ \pm}$and $K^{ \pm}$with momenta around $10 \mathrm{GeV} / \mathrm{c}$, and of $D^{*}$ mesons, which decay into $D$ mesons emitting an additional pion with a momentum around $1.6 \mathrm{GeV} / \mathrm{c}$. To achieve high mass resolution for charmed mesons and to maximize acceptance, the setup comprises a two-stage magnetic spectrometer. The first, large-angle section aims at detecting secondary particles with momenta as low as $0.5 \mathrm{GeV} / \mathrm{c}$, and the second section is optimized for secondaries above $5 \mathrm{GeV} / \mathrm{c}$. Each of the stages is equipped with high-rate scintillating fibers and micropattern gas tracking detectors for the region close to the beam up to a distance of $\sim 20 \mathrm{~cm}$, and with drift chambers, straw tubes and multiwire proportional chambers (MWPC) for large-area tracking. The spectrometer is completed with a Ring Imaging Čerenkov (RICH) detector for particle identification, electromagnetic and hadronic calorimetry, and muon detection, using drift tubes behind iron/concrete absorbers. The setup for the current phase of the experiment is shown in Fig. 1. As indicated, the small-area tracker after the first spectrometer magnet SM1 comprises 10 tracking stations for the near-beam region, each made of 2 GEM detectors with a central inactive area of $5 \mathrm{~cm}$ in diameter, with one detector rotated by $45^{\circ}$ with respect to the other. While the average particle rate per strip for a GEM detector is expected to be $20-30 \mathrm{kHz}$, the most downstream detectors will see a maximum particle rate per strip of $\sim 150 \mathrm{kHz}$ very close to the beam axis. The requirement to achieve high spatial resolution over a relatively large surface at such high radiation rates, and at the same time expose as little material as possible to the beam to reduce multiple scattering 


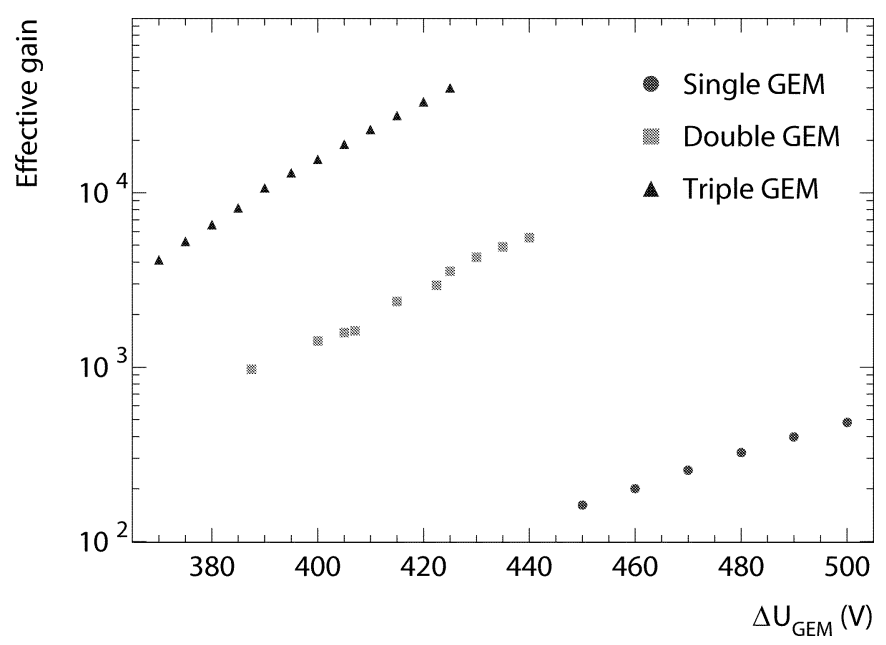

Fig. 2. Effective gain as a function of potential difference applied to each GEM foil for single, double, and triple GEM detectors.

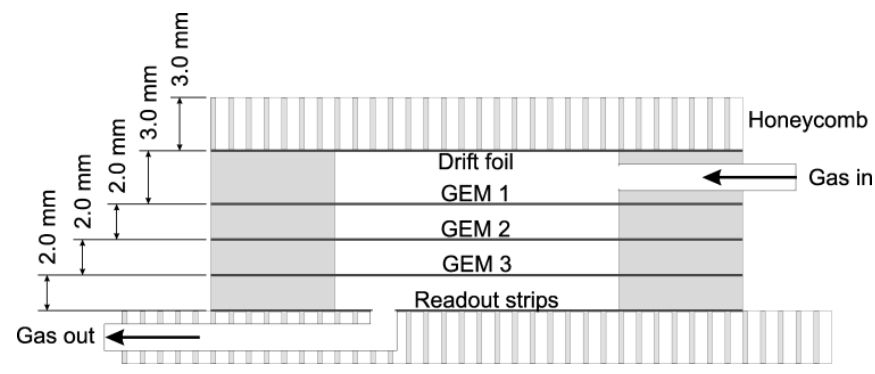

Fig. 3. Schematic cross section of a COMPASS triple GEM detector.

and secondary interactions, imposes a considerable constraint on the design of the detectors.

\section{COMPASS TRIPLE GEM DETECTORS}

The GEM (Gas Electron Multiplier) [3] is a thin (50 $\mu \mathrm{m})$ polymeric insulator foil with copper electrodes on both sides, optically semi-transparent due to a large number of holes (typically $10^{4} / \mathrm{cm}^{2}$ ) of diamater around $70 \mu \mathrm{m}$. When inserted between a drift cathode and a readout anode plate, it acts as a charge amplifier upon application of a potential difference between the two sides of the foil. In order to achieve gains suitable for efficient detection of minimum ionizing particles, several GEM amplification stages can be cascaded in a detector. Fig. 2 shows the effective gain, defined by the ratio of charge collected by the readout electrode and primary charge produced by ionizing radiation, for single, double and triple GEM devices as a function of the potential difference between the two GEM electrodes. Here, as for all following measurements, the detector was operated in a gas mixture of $70 \%$ Argon and $30 \% \mathrm{CO}_{2}$, which was chosen for its favorable properties as nonflammability, low diffusion and large drift velocity of electrons, and nonpolymerization in case of discharges.

The GEM detectors built for COMPASS consist of three multiplication stages, with a conversion gap of $3 \mathrm{~mm}$, and gaps between GEM foils and to the readout anode of $2 \mathrm{~mm}$, as shown in Fig. 3. A grid of $3 \times 3$ spacer strips of $400 \mu \mathrm{m}$ width ensures a constant separation between the GEM foils. The fact that, for a

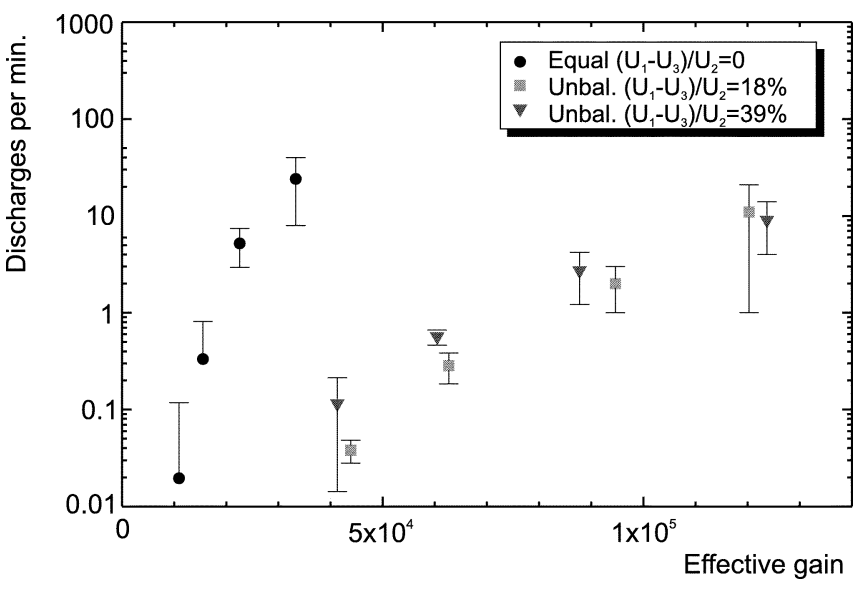

Fig. 4. Rate of $\alpha$-induced discharges for different sharings of gain between individual GEM foils.

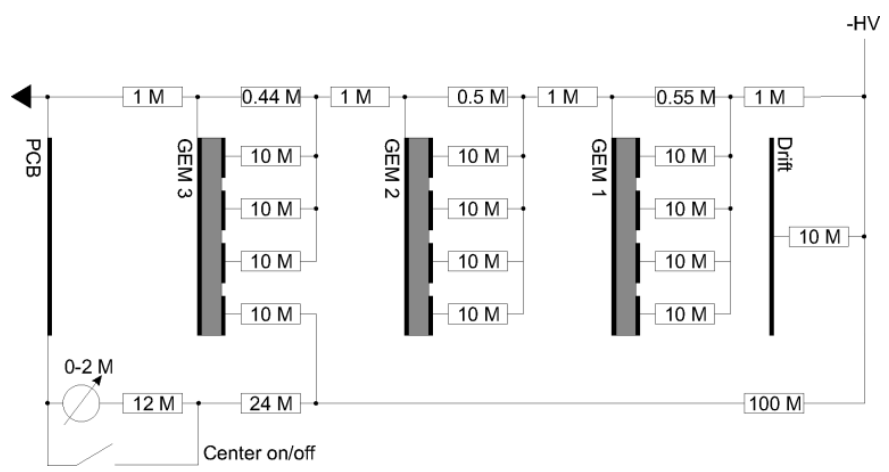

Fig. 5. Resistive voltage divider used for high voltage distribution to the various electrodes of the COMPASS triple GEM detectors. A remote-controlled switch allows to activate/deactivate the central region of $5-\mathrm{cm}$ diameter by modifying the potential drop over the last GEM in the cascade.

given gain, the triple GEM can be operated at much lower voltages over each GEM foil was found to reduce the probability of a gas discharge in presence of heavily ionizing radiation by more than one order of magnitude compared to a double GEM device [4]-[6].

Moreover, at a given gain the discharge probability under exposure to $\alpha$ particles was found to depend on the gain sharing between cascaded amplification stages, as illustrated in Fig. 4 [5], [7]. The curves show the discharge rate as a function of the total effective gain for equal voltage drops on all three GEM foils, and for $18 \%$ and $39 \%$ voltage asymmetry, defined as the difference of voltage drops over the first GEM and the third, divided by the voltage drop in the second GEM. Obviously, the discharge rate can be reduced considerably by operating the first GEM at higher gain, and successively decreasing it toward the last multiplier. In accordance with this measurement, we adopted an asymmetry of $22 \%$ for the COMPASS chambers, resulting in a reduction of discharge rate by more than one order of magnitude with respect to a symmetric gain sharing. For reasons of operational safety and material budget, all electrodes are powered from a single high voltage source through a resistive voltage divider with resistances chosen to account for the asymmetry, as shown in Fig. 5.

To reduce the energy stored in the GEM, and consequently the charge released in a discharge, the foils are segmented on 


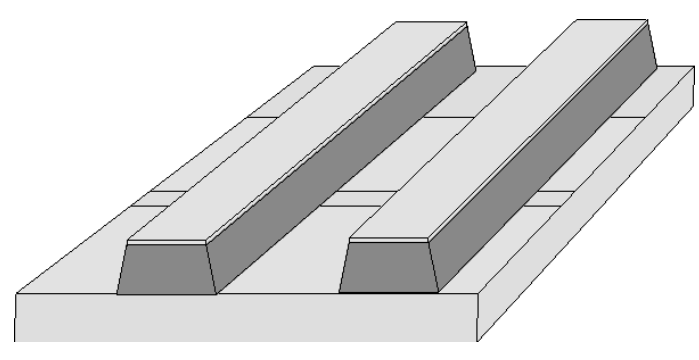

Fig. 6. Schematic view of the 2-D readout structure.

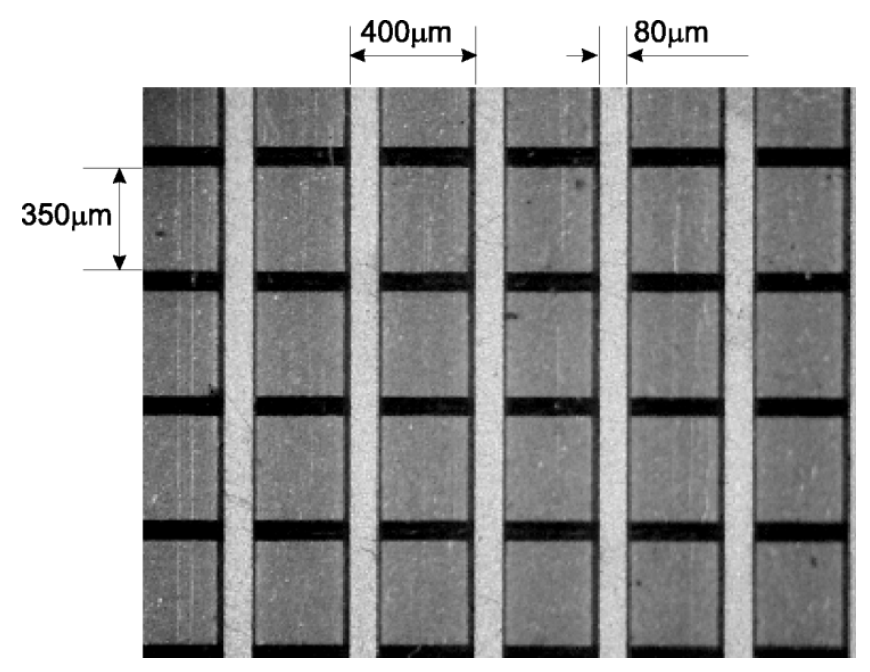

Fig. 7. Microscope photograph of the 2-D readout structure.

one side into 12 longitudinal sectors of $2.6 \times 31 \mathrm{~cm}^{2}$ size, corresponding to a capacitance per sector of $\sim 5 \mathrm{nF}$. Each sector is powered individually through high-ohmic resistors. Limiting the initial charge released in a discharge between the two GEM electrodes, where the electric field is very high, also reduces the probability, that the discharge extends all the way to the readout electrodes, below our measurement limit.

A two-dimensional (2-D) projective readout of the signal is accomplished by two orthogonal sets of 768 parallel metal strips each, the top layer of strips being insulated from the bottom one by $50-\mu$ m-thick ridges of Kapton, as shown in Fig. 6 . An equal sharing of the available charge between the two layers, as will be shown in Section $\mathrm{V}$, is achieved by adjusting the strip widths to $80 \mu \mathrm{m}$ for the top layer, and $350 \mu \mathrm{m}$ for the bottom layer, which is partly covered by the top strips. The strip pitch is 400 $\mu \mathrm{m}$ for both layers, resulting in an active area of $31 \times 31 \mathrm{~cm}^{2}$. Fig. 7 shows a microscope photograph of the readout structure, seen from the top.

The readout electronics makes use of the APV25 chip [8], a 128-channel amplifier-shaper ASIC with an analogue pipeline of 192 cells for each input channel. The chip is fabricated in a $0.25-\mu \mathrm{m}-\mathrm{CMOS}$ process to take advantage of the radiation tolerance, low noise and power consumption intrinsic to this technology. Designed for readout of silicon microstrip detectors, however, it does not have input protection against overload currents coming from the detector. Although we have significantly reduced the discharge probability and energy, we have

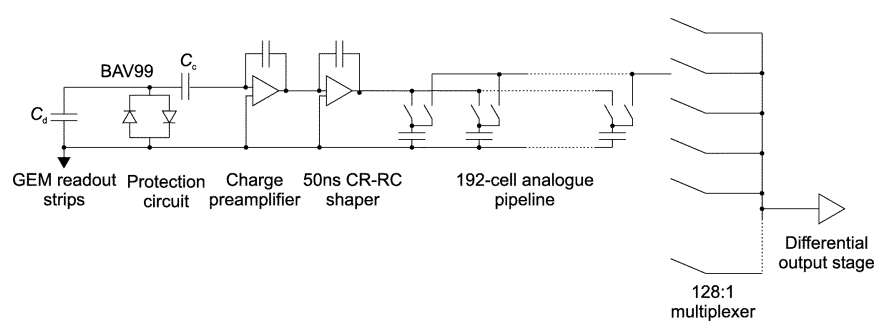

Fig. 8. Schematics of one channel of the front-end readout electronics including the protection circuit, and the APV25-S1 preamplifier-shaper, pipeline, and multiplexer stages. The protection circuit comprises a pair of inverted high-speed signal diodes (BAV99), and a coupling capacitor $\left(C_{c}=220 \mathrm{pF}\right)$. The potential of the anode strips is defined by leakage currents through the diodes, connected to ground. The capacitance of the 2-D readout structure is $C_{d} \approx 22 \mathrm{pF}$

added an external input protection circuit to maximize the reliability of the system. It consists of a pair of inverted high-speed signal diodes (BAV99) connected to ground, and a coupling capacitor $\left(C_{c}=220 \mathrm{pF}\right)$ to avoid leakage currents into the amplifier, as shown in Fig. 8. The potential of the anode strips is defined by the residual ohmic resistance of the unbiased diodes. Input signals are preamplified by a charge amplifier and shaped by a CR-RC filter with $50 \mathrm{~ns}$ peaking time. Note that only the fast electron charge is detected on the readout strips, without any slow ion component, a property of the GEM that is essential for good pile-up rejection. The amplifier outputs are sampled at a rate of $40 \mathrm{MHz}$ and stored in the pipeline, while external trigger decisions are taken. The pipeline depth allows a programmable latency of up to 160 clock cycles $(4 \mu \mathrm{s})$, the remaining locations being used to buffer the data from up to 10 events. Upon arrival of a trigger signal, up to three consecutive samples of the signal amplitude on each channel are multiplexed and read out and digitized by a 10-bit analog-to-digital converter (ADC).

\section{AgING TeSts}

The small-area tracking detectors in COMPASS are exposed to extremely high particle fluxes of $\mu$ and hadron beams, imposing considerable requirements to the radiation tolerance of their performance. It has already been demonstrated in measurements with a small $10 \times 10 \mathrm{~cm}^{2}$ double GEM prototype detector, that this kind of detector is less sensitive to aging than other micropattern gas devices [9]. To test the aging behavior of a standard COMPASS triple GEM detector, mandatory due to its different mechanical structure and larger size, we used a beam of $8.9 \mathrm{keV}$ X-rays to irradiate the detector, operating at nominal gain, for about one month at rates of up to 2.5 . $10^{4} \mathrm{~Hz} / \mathrm{mm}^{2}$ (resulting in a current density at the readout structure of $10 \mathrm{nA} / \mathrm{mm}^{2}$ ), continuously monitoring current and gain both in the exposed region and outside. Fig. 9 shows the variation of the gain, corrected for variations of ambient temperature and pressure, as a function of the collected charge [10]. The fact that no loss of gain or energy resolution was observed even after collection of more than $7 \mathrm{mC} / \mathrm{mm}^{2}$, corresponding to the total charge collected in more than seven years of operation in COMPASS, justifies the use of these detectors even in the harsh environment of the COMPASS hadron beam. 


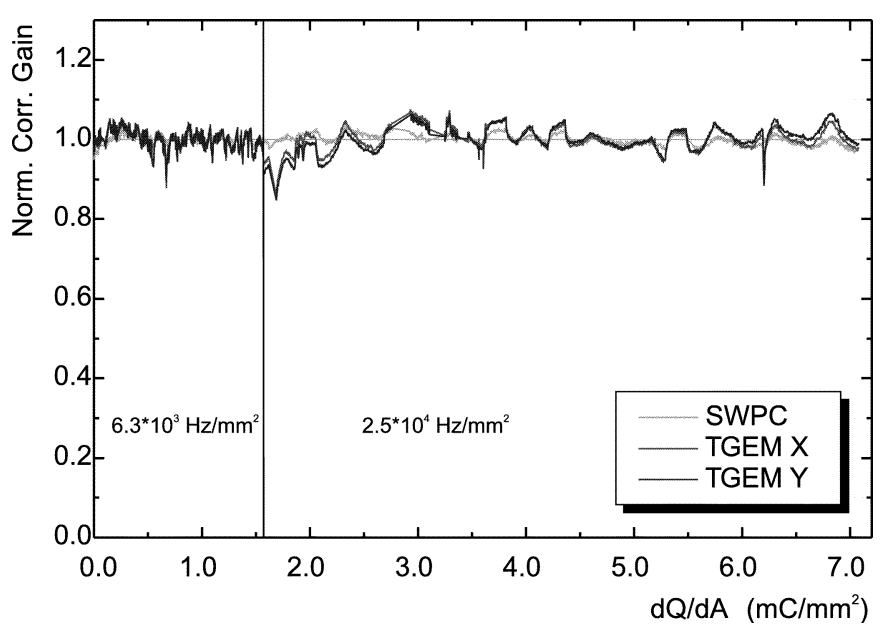

Fig. 9. Variation of the corrected gain as a function of accumulated charge. For reference measurements, a single-wire proportional counter (SWPC) was installed in the same gas line in front of the GEM detector.

\section{DeTECTOR QuAlity CONTROL}

During and after mechanical assembly of a detector, the GEM foils are tested for high voltage stability up to a potential drop of $550 \mathrm{~V}$ in a dry nitrogen atmosphere. The occurrence of spontaneous discharges or leakage currents above $5 \mathrm{nA}$ indicates a potential source of problems during a long-term operation of a detector, which has to be identified and cured. Before mounting and bonding the final front-end electronics, each chamber undergoes a standardized test and calibration procedure. To this end, the readout strips are temporarily bonded to printed circuit boards, providing access to the signal from groups of strips, which is amplified and digitized by standard laboratory electronics. A collimated source of $8.9-\mathrm{KeV} \mathrm{X}$-rays is then used to measure the gain and energy resolution for both readout coordinates on a matrix of $4 \times 4$ points on the surface of the detector. Fig. 10 shows the resulting maps of relative gain and energy resolution of a typical chamber. The observed gain fluctuations of $\sim 8 \%$ rms are attributed to local variations in the GEM hole diameter. For the positions of minimum and maximum gain an absolute gain calibration is performed by measuring the signal current on each readout plane as a function of the counting rate under irradiation with $\mathrm{X}$-rays. The resulting gain curves are displayed in Fig. 11.

\section{Performance in Particle Beams}

Several prototypes of the COMPASS GEM detectors, at various stages of their development, have been tested both in the laboratory and in particle beams [4], [6], [11]. Here, we report results obtained with two standard COMPASS triple GEM detectors, equipped with final front-end electronics, in a beam of $3.6 \mathrm{GeV} / \mathrm{c}$ protons and pions with an intensity of $10^{6}$ particles per spill from CERN's PS accelerator. Two upstream scintillation counters with an active area of $10 \times 10 \mathrm{~cm}^{2}$ in coincidence with a downstream, smaller scintillator $\left(5 \times 2 \mathrm{~cm}^{2}\right)$ provided the trigger for readout of the tracking detectors. The coincidence signal was synchronized to a 40-MHz clock and distributed to the front-end electronics. A double-sided silicon microstrip detector (active area $5 \times 7 \mathrm{~cm}^{2}$, strip pitch $50 \mu \mathrm{m}$ ) was placed

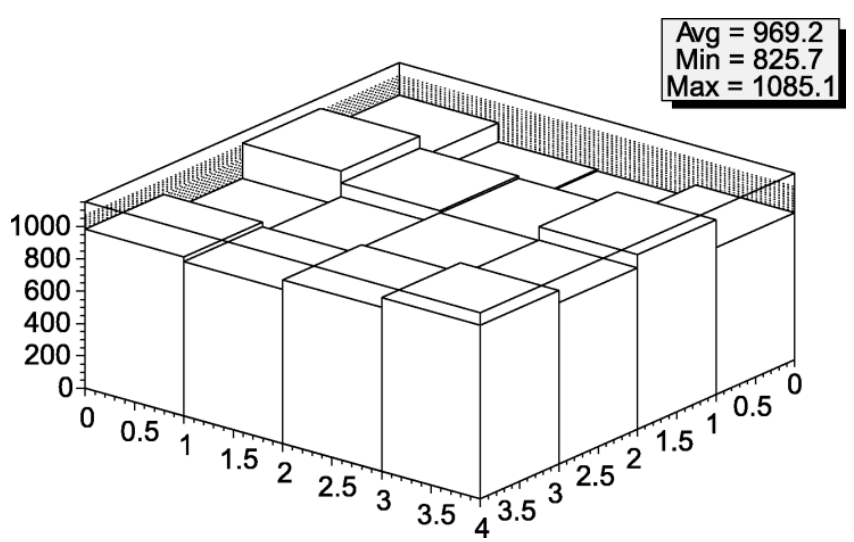

(a)

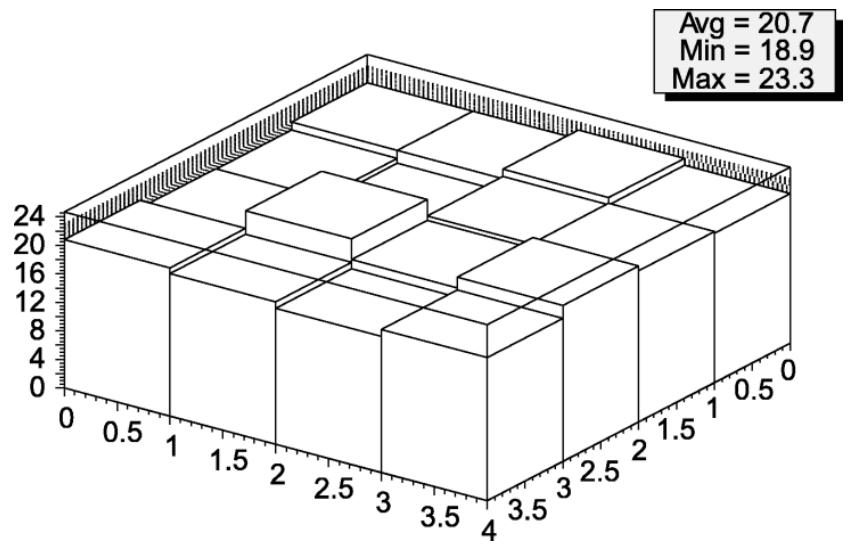

(b)

Fig. 10. (a) Maps of relative gain and (b) energy resolution measured with a collimated source of $8.9 \mathrm{keV}$ X-rays.

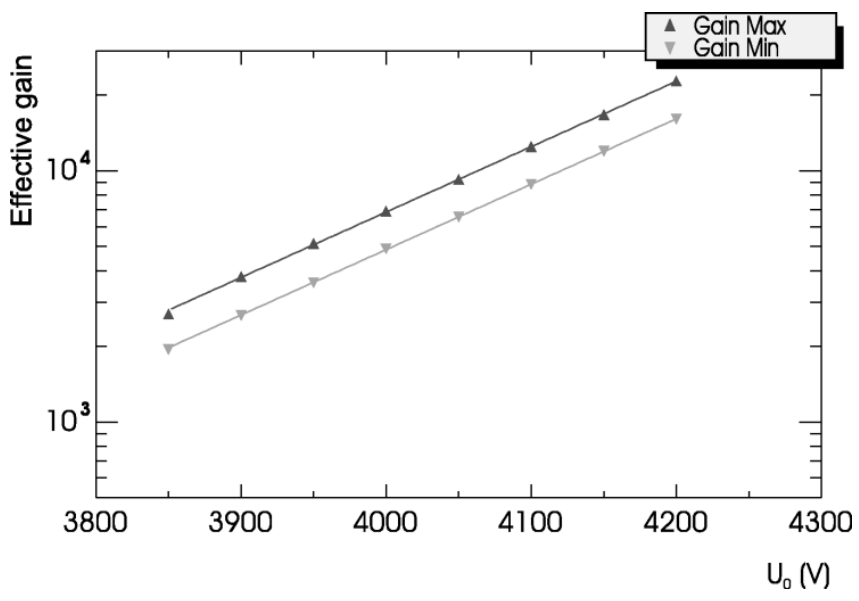

Fig. 11. Maximum and minimum effective gain for a typical COMPASS triple GEM detector as a function of the voltage applied to the resistive divider.

downstream of the GEM detectors to obtain a precise reference space point for particle tracks.

For each event, the input signal on each strip of the GEM readout structure is recorded for three consecutive time slices of $25 \mathrm{~ns}$. The trigger latency is adjusted in a way that the third sample corresponds to the peak amplitude of the signal, and the second and first samples correspond to the amplitudes 25 and 50 ns earlier, respectively. After pedestal and common mode subtraction a simple cluster finding algorithm tags all strips with 

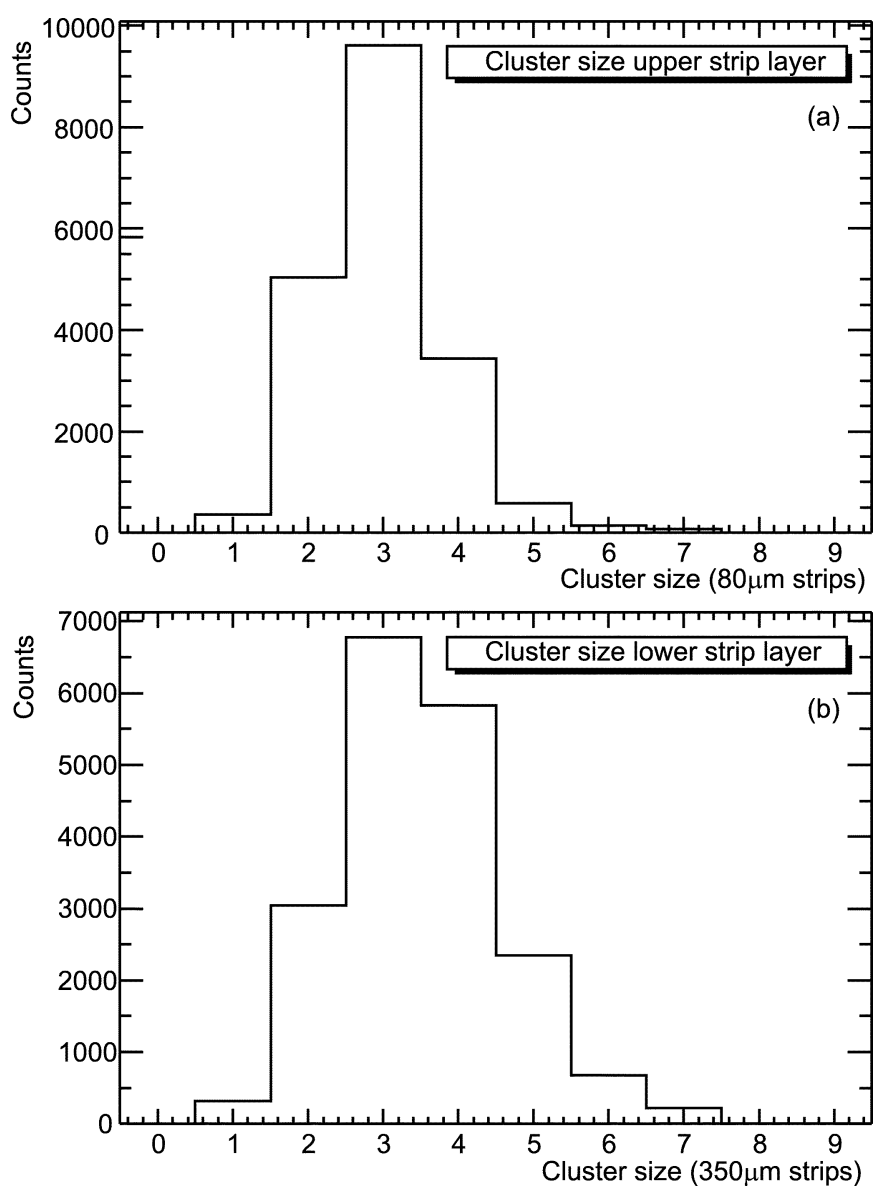

Fig. 12. Cluster size distributions for the layer of (a) $80 \mu \mathrm{m}$ wide strips and (b) $350 \mu \mathrm{m}$ wide strips.

amplitudes in the third sample exceeding a threshold of three times the strip noise, and groups together all adjacent strips fulfilling this cut. A cluster is valid if the sum of amplitudes of adjacent strips is larger than five times the cluster noise. Overlapping clusters are recognized if they are separated by a local minimum. For each cluster, the center of gravity, the width and the total charge is calculated. Fig. 12 shows the distribution of cluster sizes (number of strips above threshold) for the upper layer of strips ( $80 \mu \mathrm{m}$ width) and the lower layer ( $350 \mu \mathrm{m}$ width).

The upper and lower layer of readout strips equally share the charge cloud produced by an ionizing track, as reflected in Fig. 13, where the cluster amplitudes recorded from the upper layer of strips is plotted versus the one from the lower layer. The fact that the fraction of the signal collected on the top layer is actually larger than expected from the geometrical dimensions of the strips given in Section II, can be explained by the finite thickness of the insulating ridges between the two layers, which leads to a distortion of the electric field lines. The narrow correlation of amplitudes with a ratio of $\sim 1$ and a sigma of $\sim 0.1$ is a very important feature of the detector, which can be exploited to resolve ambiguities in the case of multi-hit events, by coupling clusters with matching signal amplitudes in both coordinates to yield space points, as illustrated in Fig. 14. Here, multiple hit events were generated offline by superposition of single hit events, and the amplitude correlation was then used in a maximum likelihood algorithm to associate hits on both pro-

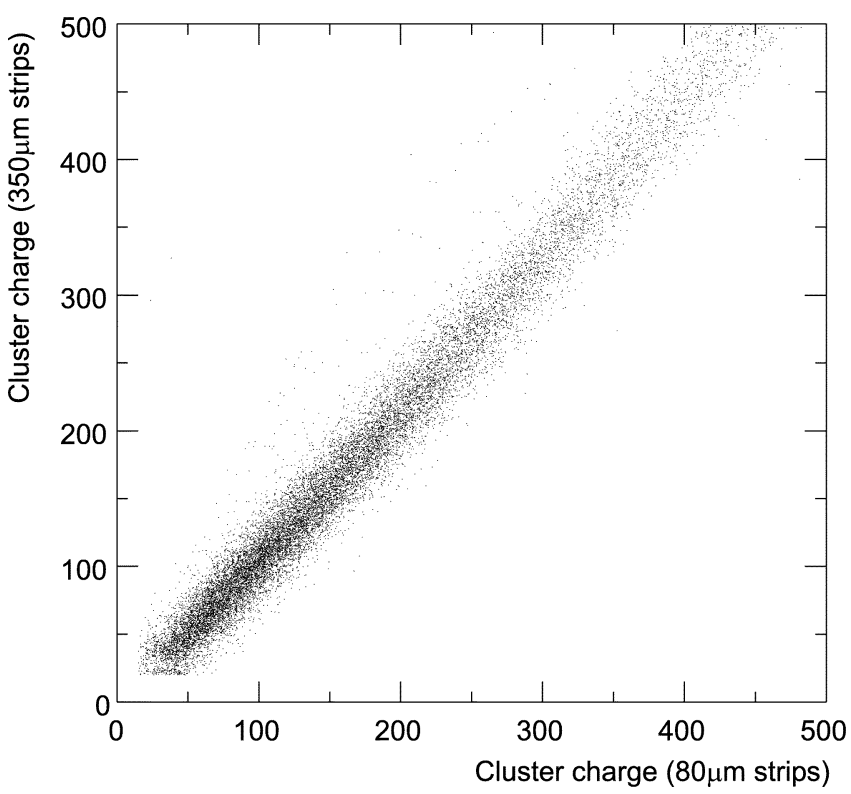

Fig. 13. Correlation of cluster amplitudes recorded on the upper ( $80 \mu \mathrm{m}$ width) and lower (350 $\mu \mathrm{m}$ width) layer of strips.

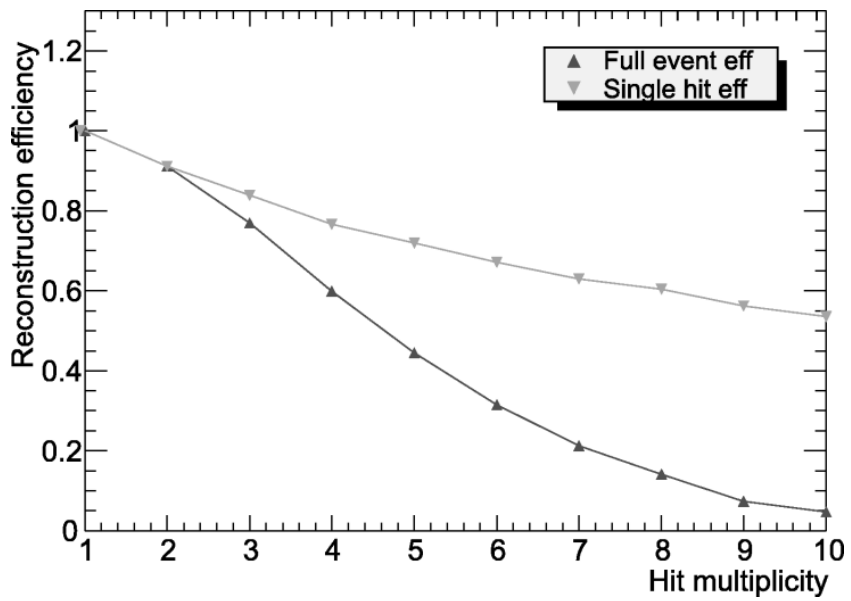

Fig. 14. Reconstruction efficiency for single hits (upper curve) and all hits in an event as a function of the hit multiplicity in the detector, using the known amplitude correlation.

jections. In a four-hit event, for example, about $80 \%$ of the clusters detected on the two projections are correctly reconstructed to space points. In $60 \%$ of the cases, even all intersection points of a 4-hit event can be fully reconstructed in a single detector, without making use of the information from other tracking detectors nearby.

Efficiency and signal-to-noise curves for detection of minimum ionizing particles were determined for both projections, and are shown in Fig. 15. Here, the efficiency is simply calculated by the ratio of the number of events with a GEM hit recorded in a small fiducial area close to the center of the detector, to the number of triggers given by the scintillating counters. Its absolute value is therefore underestimated by about $4 \%$, the fraction of inefficient regions obstructed by spacer grids and sector boundaries in this area. For this particular chamber, the plateau of full efficiency starts at a voltage of $4050 \mathrm{~V}$, corresponding to a total effective gain of $\sim 8000$, or 4000 per coor- 


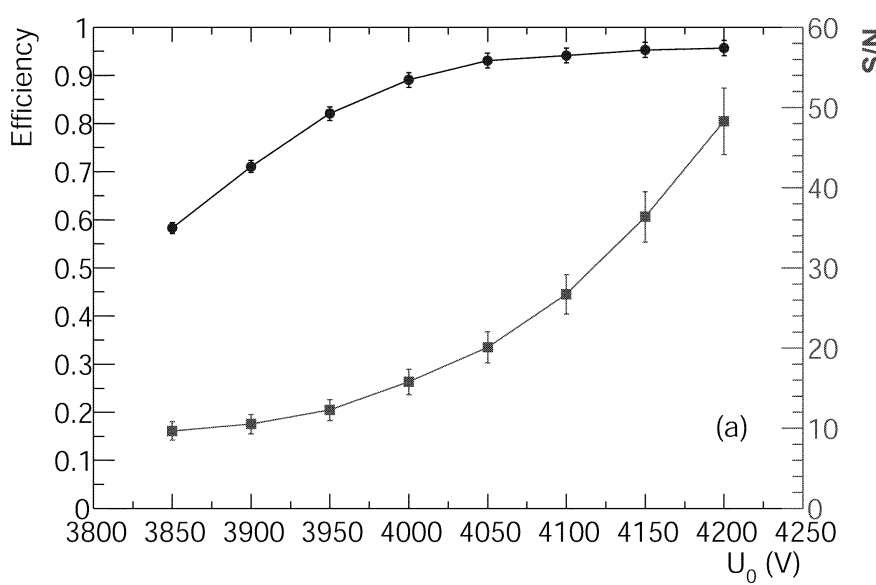

(a)

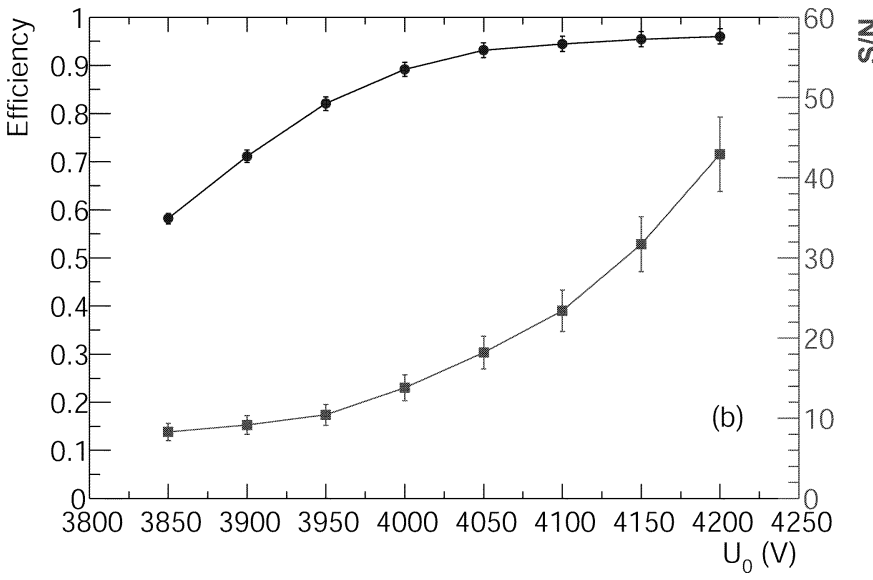

(b)

Fig. 15. Efficiency (left ordinate) for the detection of minimum ionizing particles for the (a) upper and (b) lower layer of strips, and signal-to-noise ratio (right ordinate), both as a function of the voltage applied to the resistive divider.

dinate. The signal-to-noise ratio, defined as the cluster amplitude divided by the rms cluster noise, at the beginning of the efficiency plateau is around 18.

Position-resolved characterization of a detector requires the definition of a track, which in this case was given by a space point from the first GEM detector and from the silicon microstrip detector. The efficiency versus position on the second GEM detector was then determined by counting the hits within $400 \mu \mathrm{m}$ of the expected trajectory, as displayed in Fig. 16 along the coordinate measured by the lower layer of strips. The local inefficiency at $x \approx 235 \mathrm{~mm}$ is caused by a spacer grid, the total surface of which amounts to $<1 \%$ of the total active area of the detector. Outside obstructed regions the efficiency is found to be $(99.0 \pm 0.1) \%$.

From the distribution of hit-residuals relative to the predicted hit position, shown in Fig. 17, the spatial resolution of the detector can be deduced by deconvoluting uncertainties in the tracking and effects of multiple scattering. The resulting spatial resolution is calculated to be $(46 \pm 3) \mu \mathrm{m}$.

Up to now, only the third of the three successively recorded samples of the input signal was taken into account, corresponding to the peak amplitude of the signal provided the correct timing of the trigger latency. Using in addition the information given in the second and first sample, the time of

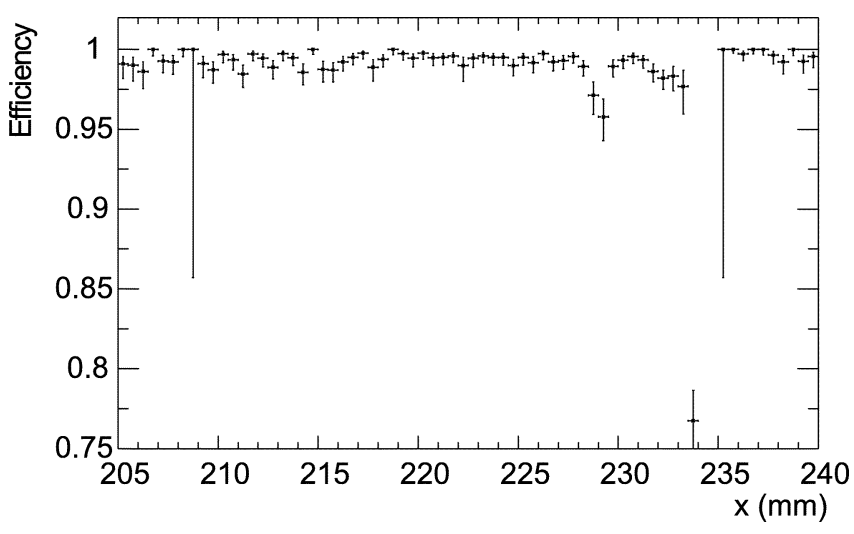

Fig. 16. Efficiency along the coordinate measured by the lower layer of strips, the local inefficiency at $x \approx 235$ showing the effect of a spacer grid.

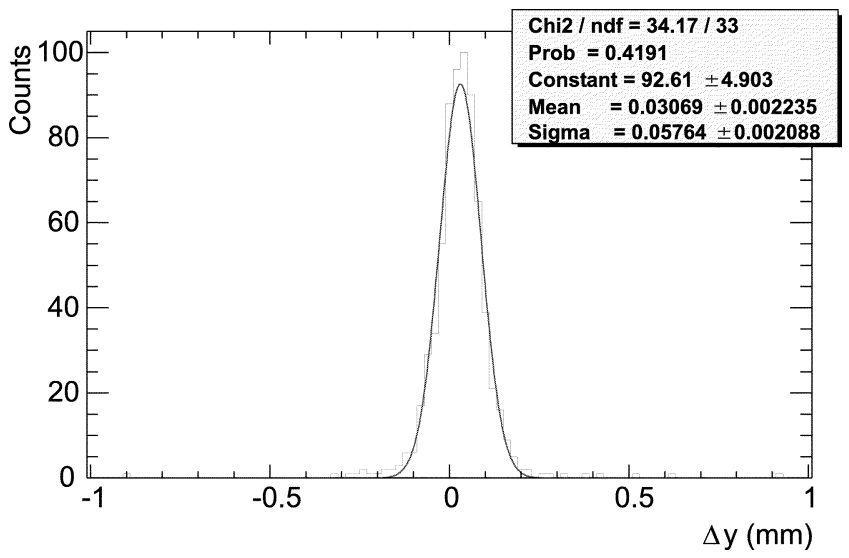

Fig. 17. Distribution of hit-residuals from the predicted track position.

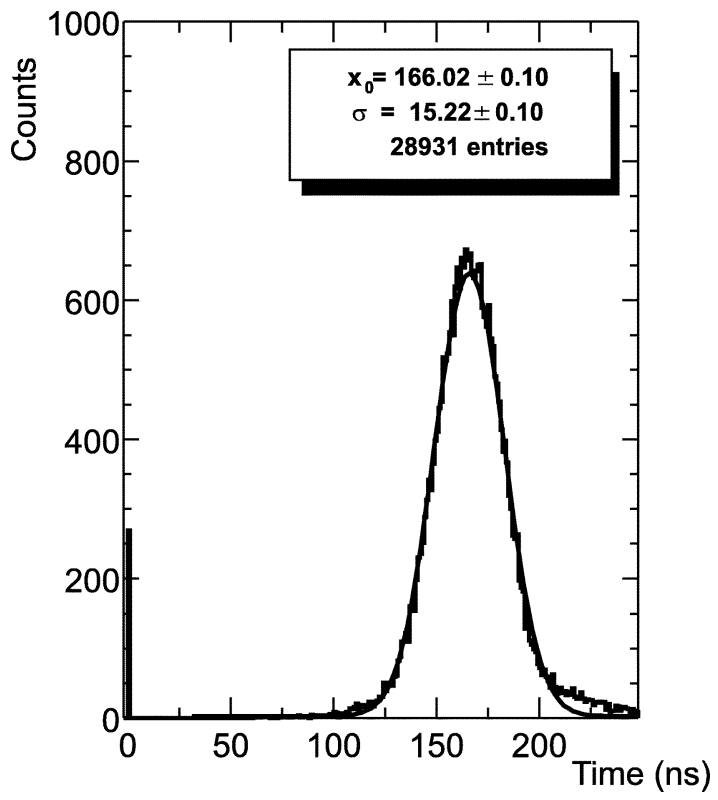

Fig. 18. Distribution of arrival times of events, derived from the signal amplitudes measured in three successive time bins of $25 \mathrm{~ns}$.

the event with respect to the trigger can be derived by fitting the (known) average response of the detector to minimum ionizing particles to the three measured samples. Fig. 18 shows the resulting distribution. Due to an unknown phase shift between 


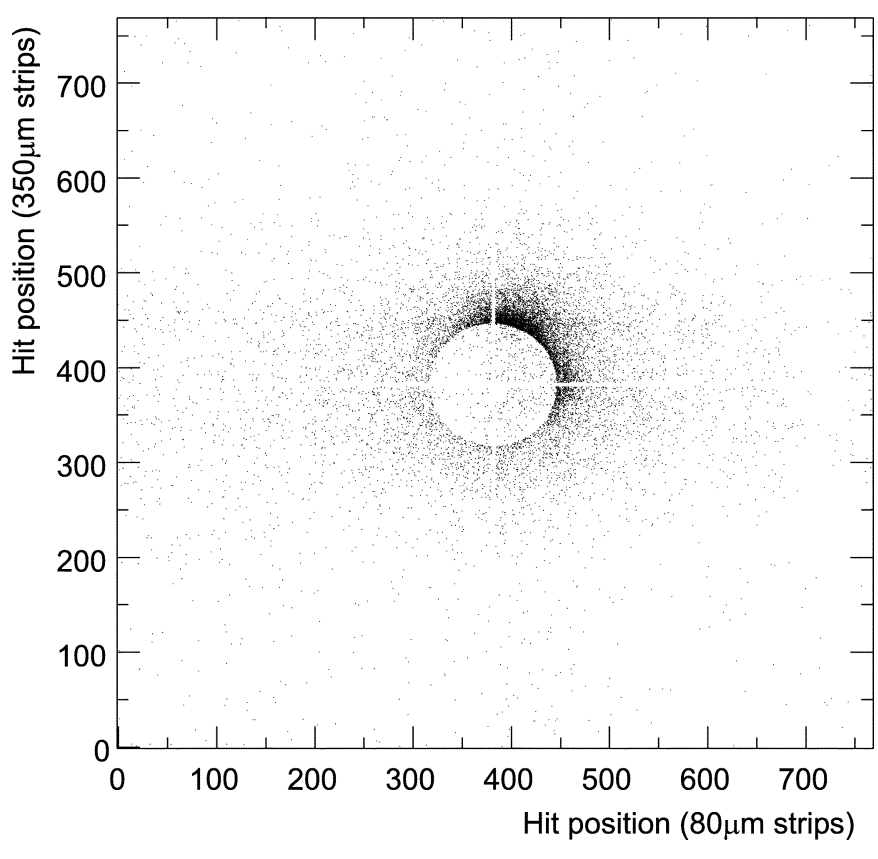

Fig. 19. Positions of maximum cluster for each event (hit map). The central sector of $5 \mathrm{~cm}$ diameter was disabled to avoid too high occupancies in the high-intensity beam of $2 \cdot 10^{8} \mu /$ spill. The cross without hits is caused by the spacer grid.

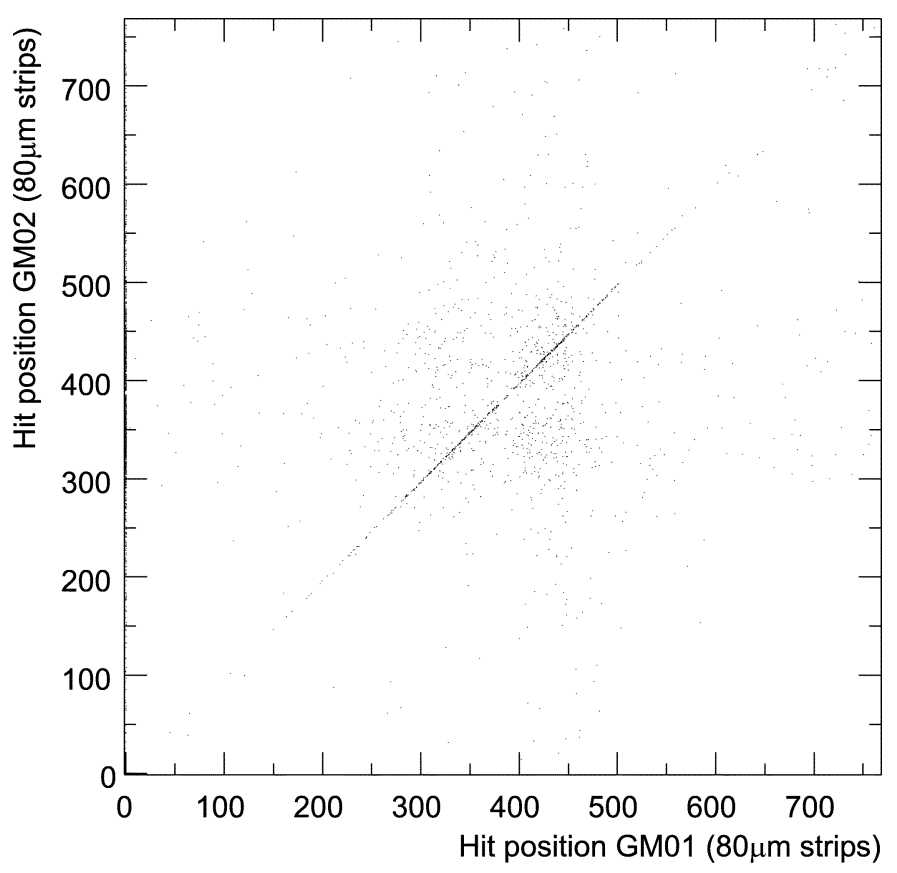

Fig. 20. Correlation of hit positions in one coordinate, measured by two detectors $0.5 \mathrm{~m}$ apart in beam direction.

the trigger signal and the $40 \mathrm{MHz}$ sampling clock, the distribution shown is the convolution of the detector time resolution (assumed Gaussian) and a 25 ns wide rectangular distribution. Deconvoluting this $25 \mathrm{~ns}$ time jitter yields a detector time resolution of $\sim 15 \mathrm{~ns}$. A measurement of the clock phase with respect to the trigger signal is being implemented in the COMPASS experiment and will remove the uncertainty in the time measurement.
After these successful tests, certifying that the detectors meet their design specifications, a total of 14 chambers were produced, installed, and commissioned in the COMPASS spectrometer for the 2001 physics run. After having been flushed with detector gas for several days and a running-in phase at moderate voltages of about one week, the detectors were set to their nominal gain, 8000 according to the results of our tests, and operated without problems at the design intensity of $2 \cdot 10^{8} \mu /$ spill. Fig. 19 shows a typical hit map from a high-intensity run, produced by plotting the positions of the maximum cluster for each event.

Fig. 20 shows a clear correlation in the position of hits measured by two different GEM detectors, $0.5 \mathrm{~m}$ apart in beam direction.

\section{SUMMARY AND OUTLOOK}

For the COMPASS small-area tracker, the first 14 triple GEM detectors out of a total of 20 have been built, tested and installed. The performance of a triple GEM detector in high radiation environments compares favorably with that of a double GEM detector in terms of discharge probability and energy. In conjunction with improvements concerning the geometry of GEM foils and the high-voltage distribution, a safe long-term operation of these devices without aging seems feasible, even in the harsh environment of a high-rate experiment like COMPASS. This was shown by accumulating a total charge of $>7 \mathrm{mC} / \mathrm{mm}^{2}$, corresponding to more than 7 years of operation in COMPASS, without any loss of gain or energy resolution. The operational characteristics of these devices have been studied in various test beams. Fully efficient ( $>98 \%$ ) detection of minimum ionizing particles on both coordinates of the 2-D readout was achieved at a gain of $\sim 8000$, corresponding to an SNR of $\sim 18$. With a spatial resolution better than $50 \mu \mathrm{m}$, and a time resolution around $15 \mathrm{~ns}$, these devices were proven to have excellent tracking properties. A rate capability in excess of $10^{5} \mathrm{~Hz} / \mathrm{mm}^{2}$, and an outstanding multi-hit resolution due to their 2-D readout make these detectors well suited for exposure to high particle rates. Their good performance during eight weeks in the high-intensity muon beam of COMPASS in 2001 confirmed that GEM detectors meet the requirements for tracking devices in modern high energy physics experiments.

\section{REFERENCES}

[1] "COMPASS: A proposal for a common muon and proton apparatus for structure and spectroscopy," The COMPASS Collaboration, CERN/SPSLC 96-14, SPSC/P 297, 1996.

[2] G. K. Mallot, "Accessing the gluon polarization in deep inelastic muon scattering," Progr. Part. Nucl. Phys., vol. 36, pp. 39-48, 1996.

[3] F. Sauli, "GEM: A new concept for electron amplification in gas detectors," Nucl. Instr. Meth., vol. A386, pp. 531-534, 1997.

[4] S. Bachmann, A. Bressan, B. Ketzer, M. Deutel, L. Ropelewski, F. Sauli, A. Bondar, A. Buzulutskov, L. Shekhtman, A. Sokolov, A. Tatarinov, A. Vasil'ev, S. Kappler, and E. Schulte, "Performance of GEM detectors in high intensity particle beams," Nucl. Instr. Meth, vol. A470, pp. 548-561, 2001.

[5] S. Bachmann, A. Bressan, M. Capeáns, M. Deutel, S. Kappler, B. Ketzer, A. Polouektov, L. Ropelewski, F. Sauli, E. Schulte, L. Shekhtman, and A. Sokolov, "Discharge studies and prevention in the gas electron multiplier (GEM)," Nucl. Instr. Meth., vol. A479, pp. 294-308, 2002. 
[6] B. Ketzer, S. Bachmann, M. Capeáns, M. Deutel, J. Friedrich, S. Kappler, I. Konorov, S. Paul, A. Placci, K. Reisinger, L. Ropelewski, L. Shekhtman, and F. Sauli, "GEM detectors for COMPASS," IEEE Trans. Nucl. Sci., vol. 48, pp. 1065-1069, 2001.

[7] C. Altunbas, M. Capeáns, K. Dehmelt, J. Ehlers, J. Friedrich, I. Konorov, A. Gandi, S. Kappler, B. Ketzer, R. De Oliveira, S. Paul, A. Placci, L. Ropelewski, F. Sauli, F. Simon, and M. van Stenis, "Construction, test and commissioning of the triple-GEM tracking detectors for COMPASS," Nucl. Instr. Meth. A, vol. 490, pp. 177-203, 2002.

[8] L. L. Jones, M. J. French, Q. Morrissey, A. Neviani, M. Raymond, G. Hall, P. Moreira, and G. Cervelli, "The APV25 deep submicron readout chip for CMS detectors," in Proc. 5th Conf. Electronics for LHC Experiments, 1999, CERN-99-09, pp. 162-166.
[9] S. Bachmann, A. Bressan, A. Placci, L. Ropelewski, and F. Sauli, "Development and test of large size GEM detectors," IEEE Trans. Nucl. Sci., vol. 47, pp. 1412-1415, 2000.

[10] M. C. Altunbas, K. Dehmelt, S. Kappler, B. Ketzer, L. Ropelewski, and F. Sauli, "Aging measurements with the gas electron multiplier (GEM)," in Proc. Int. Workshop on Aging Phenomena in Gaseous Detectors, Hamburg, 2001.

[11] A. Bressan, J. C. Labbé, P. Pagano, L. Ropelewski, and F. Sauli, "Beam tests of the gas electron multiplier," Nucl. Instr. Meth., vol. A425, pp. 262-276, 1999. 